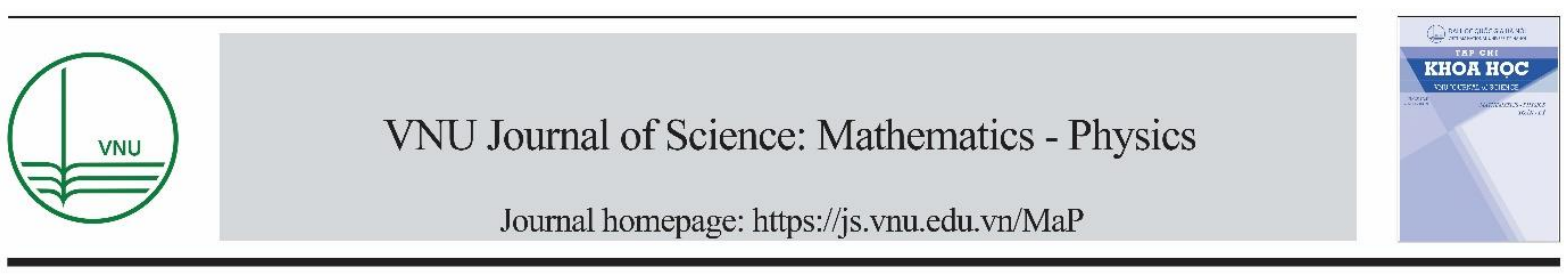

\title{
The Buckling of Three-phase Orthotropic Composite Plates Used in Composite Shipbuilding
}

\author{
Pham Van Thu* \\ Nha Trang University, 44 Hon Ro, Phuoc Dong, Nha Trang, Khanh Hoa, Vietnam
}

Received 20August 2018

Revised 27December 2018; Accepted 31December 2018

\begin{abstract}
This paper presents the investigation on the buckling of three-phase orthotropic composite plates used in shipbuilding subjected to mechanical loads by analytical approach. The basic equations are established based on the Classical Plate Theory. The analytical method is used to obtain the expressions of critical loads of the three-phase composite plate. The results in the article are compared to the results obtained by other authors to validate the reliability of the present method. The effects of fiber and particle volume fraction, material and geometrical parameters on the critical load of three-phase composite plates are discussed in detail.
\end{abstract}

Keywords: Buckling, three-phase composite, critical load, orthotropic plate, composite shipbuiding.

\section{Introduction}

Composite is a material composed of two or more component materials to obtain better properties compared to other regular materials $[1,2]$. Therefore, composite materials are widely used in all fields: power, aviation, construction, shipbuilding, civil and medical fields....

In addition to advantages of composite material such as: nonreactive with environment, lightweight, durable in corrosive environment, it also has disadvantages: easily permeable, flammable features $[2,3]$ and low level of hardness.

In the shipbuilding industry, nowadays small and medium-sized patrol boats, cruise ships, and fishing boats are mainly made from composite material. In order to increase the waterproofing, fireretardancy and the hardness of the material, besides the fiber reinforcement usually added reinforced particles to the reinforced polymer matrix $[4,5]$. In fact, there are actually three-phase composites: polymer matrix, reinforced fiber and particles.

\footnotetext{
* Tel.: 84-914005180.

Email: phamvan.thu70@gmail.com

https//doi.org/ 10.25073/2588-1124/vnumap.4313
} 
Adding reinforced particles to the polymer matrix, the mechanical properties of plate and its shell structure will vary (effect on tensile, bending and impact strength) [5]. Therefore, it is necessary to control the effect of the ratio of component phases to the durability of the structure while still meeting the desired criteria such as waterproofing or fire-retardancy [6-8].

Plate, shell and panel are the basic structures in engineering and manufacturing industry. These structures play an important role in mainly supporting all structures of machinery and equipment. Buckling of composite plate and shell is first and foremost issue in optimal design. In fact, many researchers are interested in this issue [6-13]. Therefore, research on three-phase composite plate and shell is crucial in both science and practice.

In fact, most composite materials currently used in shipbuilding have a orthotropic configuration. The paper introduces a study on the buckling of three-phase orthotropic composite plates used in shipbuilding by analytical methods. This paper approaches in the direction of critical load expressions. The effect of fiber, particle, material and geometry characteristics on the critical load of composite threephase plates is discussed in detail. The results calculated according to the approach in the paper, compared with the results obtained by other authors in the possible cases to test the reliability of the method.

\section{Determining elastic modulus of three-phase composite}

Three-phase composite has been proposed for study and solve scientific problems posed by methods in $[6,7,14-16]$, i.e. solved step by step in a two-phase model from the point of view described by the formula:

$$
1 D_{m}=O_{m}+1 D
$$

First step: considering 2-phase composite including: initial matrix phase and filling particles, composite are considered identical, isotropic and have 2 elastic coefficients. The elastic coefficients of the $\mathrm{O}_{\mathrm{m}}$ composite are now called composite assumptions.

Second step: determining the elastic coefficients of composite between the assumed matrix and reinforced fibers.

Assuming composite components (matrix, fiber, particle) are all identical, isotropic, then we will denote $\mathrm{E}_{\mathrm{m}}, \mathrm{G}_{\mathrm{m}}, v_{\mathrm{m}}, \psi_{\mathrm{m}} ; \mathrm{E}_{\mathrm{a}}, \mathrm{G}_{\mathrm{a}}, v_{\mathrm{a}}, \psi_{\mathrm{a}} ; \mathrm{E}_{\mathrm{c}}, \mathrm{G}_{\mathrm{c}}, v_{\mathrm{c}}, \psi_{\mathrm{c}}$ respectively as elastic modulus, Poisson's ratio and component ratio (according to volume) of matrix, fiber, particle. From here on, matrix-related quantities will be written with the index $\mathrm{m}$; fiber-related quantities will have index a and index c for particle. According to Vanin and Duc [17], elastic modules of assumed composite are received as follows:

$$
\begin{gathered}
\bar{G}=G_{m} \frac{1-\psi_{c}\left(7-5 v_{m}\right) H}{1+\psi_{c}\left(8-10 v_{m}\right) H} \\
\bar{K}=K_{m} \frac{1+4 \psi_{c} G_{m} L\left(3 K_{m}\right)^{-1}}{1-4 \psi_{c} G_{m} L\left(3 K_{m}\right)^{-1}}
\end{gathered}
$$

With:

$$
L=\frac{K_{c}-K_{m}}{K_{c}+\frac{4 G_{m}}{3}}, \quad H=\frac{G_{m} / G_{c}-1}{8-10 v_{m}+\left(7-5 v_{m}\right) \frac{G_{m}}{G_{c}}}
$$


$\overline{\mathrm{G}}, \overline{\mathrm{K}}$ : Shear elastic modulus và bulk modulus of assumed matrix.

$\bar{E}, \bar{v}$ are calculated from $\bar{K}, \bar{G}$ as follows:

$$
\bar{E}=\frac{9 \bar{K} \bar{G}}{3 \bar{K}+\bar{G}}, \quad \bar{v}=\frac{3 \bar{K}-2 \bar{G}}{6 \bar{K}-2 \bar{G}}
$$

The elastic modulus of three-phase fiber-reinforced composite selected is determined according to the formulas of Vanin [18] with 6 independent coefficients as follows:

$$
\begin{aligned}
& E_{11}=\psi_{a} E_{a}+\left(1-\psi_{a}\right) \bar{E}+\frac{8 \bar{G} \psi_{a}\left(1-\psi_{a}\right)\left(v_{a}-\bar{v}\right)}{2-\psi_{a}+\bar{\chi} \psi_{a}+\left(1-\psi_{a}\right)\left(\chi_{a}-1\right) \frac{\bar{G}}{G_{a}}} \\
& E_{22}=\left\{\frac{v_{21}^{2}}{E_{11}}+\frac{1}{8 \bar{G}}\left[\frac{2\left(1-\psi_{a}\right)(\bar{\chi}-1)+\left(\chi_{a}-1\right)\left(\bar{\chi}-1+2 \psi_{a}\right) \frac{\bar{G}}{G_{a}}}{2-\psi_{a}+\bar{\chi} \psi_{a}+\left(1-\psi_{a}\right)\left(\chi_{a}-1\right) \frac{\bar{G}}{G_{a}}}+2 \frac{\bar{\chi}\left(1-\psi_{a}\right)+\left(1+\psi_{a} \bar{\chi}\right) \frac{\bar{G}}{G_{a}}}{\bar{\chi}+\psi_{a}+\left(1-\psi_{a}\right) \frac{\bar{G}}{G_{a}}}\right]\right\}^{-1} \\
& G_{12}=\bar{G} \frac{1+\psi_{a}+\left(1-\psi_{a}\right) \frac{\bar{G}}{G_{a}}}{1-\psi_{a}+\left(1+\psi_{a}\right) \frac{\bar{G}}{G_{a}}} ; \quad G_{23}=\bar{G} \frac{\bar{\chi}+\psi_{a}+\left(1-\psi_{a}\right) \frac{\bar{G}}{G_{a}}}{\left(1-\psi_{a}\right) \bar{\chi}+\left(1+\bar{\chi} \psi_{a}\right) \frac{\bar{G}}{G_{a}}} \\
& v_{23}=-\frac{E_{22} v_{21}^{2}}{E_{11}}+\frac{E_{22}}{8 \bar{G}}\left[2 \frac{\left(1-\psi_{a}\right) \bar{\chi}+\left(1+\psi_{a} \bar{\chi}\right) \frac{\bar{G}}{G_{a}}}{\bar{\chi}+\psi_{a}+\left(1-\psi_{a}\right) \frac{\bar{G}}{G_{a}}}-\frac{2\left(1-\psi_{a}\right)(\bar{\chi}-1)+\left(\chi_{a}-1\right)\left(\bar{\chi}-1+2 \psi_{a}\right) \frac{\bar{G}}{G_{a}}}{2-\psi_{a}+\bar{\chi} \psi_{a}+\left(1-\psi_{a}\right)\left(\chi_{a}-1\right) \frac{\bar{G}}{G_{a}}}\right] \\
& v_{21}=\bar{v}-\frac{(\bar{\chi}+1)\left(\bar{v}-v_{a}\right) \psi_{a}}{2-\psi_{a}+\bar{\chi} \psi_{a}+\left(1-\psi_{a}\right)\left(\chi_{a}-1\right) \frac{\bar{G}}{G_{a}}}
\end{aligned}
$$

With $\bar{\chi}=3-4 \bar{v} ; \chi_{a}=3-4 v_{a}$

\section{Governing equations}

The main differential equation for buckling analysis of orthotropic plates (Appendix A) is:

$$
\begin{aligned}
& D_{11} \frac{\partial^{4} w_{0}}{\partial \mathrm{x}^{4}}+2\left(D_{12}+2 D_{66}\right) \frac{\partial^{4} w_{0}}{\partial x^{2} \partial y^{2}}+D_{22} \frac{\partial^{4} w_{0}}{\partial \mathrm{y}^{4}} \\
& =N_{x} \frac{\partial^{2} w_{0}}{\partial x^{2}}+2 N_{x y} \frac{\partial^{2} w_{0}}{\partial x \partial y}+N_{y} \frac{\partial^{2} w_{0}}{\partial y^{2}}+q
\end{aligned}
$$




\subsection{The buckling of three-phase orthotropic plate subjected to biaxial compression}

In case, a rectangular orthotropic plate is subjected to a uniform compression on each edge with the respective force of $N_{x}=-N_{0}$ and $N_{y}=-\beta N_{0}$, without horizontal load (7) becomes:

$$
D_{11} \frac{\partial^{4} w_{0}}{\partial \mathrm{x}^{4}}+2\left(D_{12}+2 D_{66}\right) \frac{\partial^{4} w_{0}}{\partial x^{2} \partial y^{2}}+D_{22} \frac{\partial^{4} w_{0}}{\partial \mathrm{y}^{4}}=-N_{0} \frac{\partial^{2} w_{0}}{\partial x^{2}}-\beta N_{0} \frac{\partial^{2} w_{0}}{\partial y^{2}}
$$

Where:

$\mathrm{w}_{0}$ : is displacement in $\mathrm{z}$ direction of the plate

$\mathrm{N}_{0}$ : Axial compressive force per 1 unit of plate's length.

$D_{i j}(i, j=1,2,6)$ : is the bending stiffness of the plate.

$\left(Q_{i j}^{\prime}\right)_{k}$ : Hardness coefficient of the $\mathrm{k}^{\text {th }}$ layer.

$\mathrm{Z}_{\mathrm{k}}$ : is the distance from the middle surface of the plate to the bottom of the $\mathrm{k}^{\text {th }}$ layer.

In this study, the edges of composite plates are assumed to be single supported:

$$
\begin{aligned}
& \text { At } x=0 \text { and } x=a: w_{0}=M_{x}=0 ; \\
& \text { At } y=0 \text { and } y=b: w_{0}=M_{y}=0 ;
\end{aligned}
$$
form:

The boundary conditions (9) and (10) are always satisfied when the deflection function is in the

$$
\mathrm{w}_{0}(\mathrm{x}, \mathrm{y})=\mathrm{A}_{\mathrm{mn}} \sin \frac{\mathrm{m} \pi \mathrm{x}}{\mathrm{a}} \sin \frac{\mathrm{n} \pi \mathrm{y}}{\mathrm{b}}
$$

Introducing (11) into (8) and solve the equation for the following solution:

$$
N_{0}=\frac{\pi^{2}\left[D_{11} m^{4}+2\left(D_{12}+2 D_{66}\right) m^{2} n^{2} R^{2}+D_{22} n^{4} R^{4}\right]}{a^{2}\left(m^{2}+\beta n^{2} R^{2}\right)}
$$

Where:

$R=a / b:$ ratio of length / width of plate

$$
\left.\begin{array}{c}
D_{11}=\left[\left(R_{Q}-1\right) \alpha+1\right] \frac{Q_{11} e^{3}}{12}=\left[\left(R_{Q}-1\right) \alpha+1\right] \frac{e^{3}}{12} \frac{E_{11}}{1-v_{12}^{2} R_{Q}} \\
D_{12}=\frac{Q_{12} e^{3}}{12}=\frac{e^{3}}{12} \frac{v_{12} E_{22}}{1-v_{12}^{2} R_{Q}} ; \quad v_{12}=v_{21} \frac{1}{R_{Q}} \\
D_{22}=\left[\left(1-R_{Q}\right) \alpha+R_{Q}\right] \frac{Q_{11} e^{3}}{12}=\left[\left(1-R_{Q}\right) \alpha+R_{Q}\right] \frac{e^{3}}{12} \frac{E_{11}}{1-v_{12}^{2} R_{Q}} \\
\alpha=\frac{1}{\left(1+R_{e}\right)^{3}}+\frac{R_{e}(n-3)\left[R_{e}(n-1)+2(n+1)\right]}{\left(n^{2}-1\right)\left(1+R_{e}\right)^{3}}
\end{array}\right\}
$$


$R_{Q}=E_{22} / E_{11}:$ ratio Young's modulus

$R_{e}=e_{0} / e_{90}$ : ratio of total thickness of layer $0^{\circ} /$ total thickness of layer $90^{\circ}$.

$e=e_{0}+e_{90}$ : thickness of plate.

n: number of layers (only for formula no. 13) . (6).

$E_{11}, E_{22}, v_{21}, G_{12}$ : are the coefficients of three-phase composite material determined by the formula

Put expressions $E_{11}, E_{22}, v_{21}, G_{12}$ in (6) into (13), then put into expression (12), We get the $\mathrm{N}_{0}$ force value depending on $\psi_{\mathrm{a}}, \psi_{\mathrm{c}}, \mathrm{a} / \mathrm{b}$ and $\mathrm{e}$, respectively the volume ratio of fiber, particle and geometric dimensions of plates:

$$
\begin{aligned}
& N_{0}=N_{\left(\psi_{a}, \psi_{c}, a / b, e\right)} \\
= & \frac{\pi^{2}\left[\left(P_{1}+1\right) P_{2} m^{4}+2\left(v_{21} P_{2}+\frac{e^{3}}{6} G_{12}\right) m^{2} n^{2} R^{2}+\left(\frac{E_{22}}{E_{11}}-P_{1}\right) P_{2} n^{4} R^{4}\right]}{a^{2}\left(m^{2}+\beta n^{2} R^{2}\right)}
\end{aligned}
$$

Where:

Put: $P_{1}=\left(R_{Q}-1\right) \alpha=\left(\frac{E_{22}}{E_{11}}-1\right) \alpha$ and $P_{2}=\frac{e^{3}}{12} \frac{E_{11}}{1-v_{12}^{2} R_{Q}}=\frac{e^{3}}{12} \frac{E_{11}}{1-v_{12}^{2} \frac{E_{22}}{E_{11}}}$

The equation (14) is the basic equation with the variables: $\psi_{\mathrm{a}}, \psi_{\mathrm{c}}, a / b$ and e used to study the buckling of the three-phase orthotropic plate under biaxial compression.

The critical force corresponds to the values of $\mathrm{m}$ and $\mathrm{n}$ making $\mathrm{N}_{\mathrm{o}}$ smallest. With $m=n=1$, the expression (14) becomes:

$$
N_{t h}(1,1)=\frac{\pi^{2}\left[\left(P_{1}+1\right) P_{2}+2\left(v_{21} P_{2}+\frac{e^{3}}{6} G_{12}\right) R^{2}+\left(\frac{E_{22}}{E_{11}}-P_{1}\right) P_{2} R^{4}\right]}{a^{2}\left(1+\beta R^{2}\right)}
$$

\subsection{The buckling of the three-phase orthotropic plate subjected to axial compression}

When the plate is compressed in $\mathrm{x}$ direction, then $\beta=0$ and (14) becomes:

$$
=\frac{\pi^{2}\left[\left(P_{1}+1\right) P_{2} m^{4}+2\left(v_{21} P_{2}+\frac{e^{3}}{6} G_{12}\right) m^{2} n^{2} R^{2}+\left(\frac{E_{22}}{E_{11}}-P_{1}\right) P_{2} n^{4} R^{4}\right]}{m^{2} a^{2}}
$$

The equation (16) is the equation with the variables: $\psi_{\mathrm{a}}, \psi_{\mathrm{c}}, \mathrm{a} / \mathrm{b}$ and e used to study the buckling of the three-phase orthotropic plate bearing axial compression.

The smallest value of $\mathrm{N}_{0}$ corresponding to $n=1$ at $\mathrm{R}=[\mathrm{m}(\mathrm{m}+1)]^{1 / 2}\left(\frac{\mathrm{P}_{1}+1}{\frac{\mathrm{E}_{22}}{\mathrm{E}_{11}}-\mathrm{P}_{1}}\right)^{1 / 4}$ is:

$$
N_{t h}(m, 1)=\frac{\pi^{2}\left[\left(P_{1}+1\right) P_{2} m^{4}+2\left(v_{21} P_{2}+\frac{e^{3}}{6} G_{12}\right) m^{2} R^{2}+\left(\frac{E_{22}}{E_{11}}-P_{1}\right) P_{2} R^{4}\right]}{m^{2} a^{2}}
$$




\section{Results and discussion}

Survey of three-phase composite plate of axb dimensions, made from AKA resin, WR800 glass cloth and $\mathrm{TiO}_{2}$ particle including 07 layers $0^{\circ}$ and $90^{\circ}$ in the order of layers notated $7(90 / 0) \equiv[90 / 0 / 90 / 0 / 90 / 0 / 90]$ and $7(0 / 90) \equiv[0 / 90 / 0 / 90 / 0 / 90 / 0]$, the plate is composed of the following component materials:

$$
\begin{array}{lccl}
\text { AKA resin } & : & \mathrm{E}_{\mathrm{m}}=1.43 \mathrm{GPa} & ; v_{\mathrm{m}}=0.345 \\
\text { Glass reinforced fiber : } & \mathrm{E}_{\mathrm{a}}=22.0 \mathrm{Gpa} & ; v_{\mathrm{a}}=0.24 \\
\mathrm{TiO}_{2} \text { particle } & : & \mathrm{E}_{\mathrm{c}}=5.58 \mathrm{Gpa} & ; v_{\mathrm{c}}=0.20
\end{array}
$$

Replace the values (18) into the formula (15) to have results shown in the following tables:

\begin{tabular}{|c|c|c|c|c|c|c|c|c|c|c|}
\hline \multicolumn{11}{|c|}{$\psi_{\mathrm{c}}=0.2$ (constant particle ratio) - Laminated plates $7(90 / 0)$, with $\beta=1, \mathrm{~b}=0.4 \mathrm{~m}$ and $\mathrm{m}=\mathrm{n}=1$. } \\
\hline $\begin{array}{l}\Psi_{\mathrm{a}} \\
(\%)\end{array}$ & $\begin{array}{l}\mathrm{E}_{11} \\
\text { (Gpa) }\end{array}$ & $\begin{array}{l}\mathrm{E}_{22} \\
\text { (Gpa) }\end{array}$ & $\mathrm{R}_{\mathrm{e}}$ & $\begin{array}{l}\mathrm{D}_{11} \\
\left(\mathrm{~Pa}^{3} \mathrm{~m}^{3}\right)\end{array}$ & $\begin{array}{l}\mathrm{D}_{12} \\
\left(\mathrm{~Pa}^{3} \mathrm{~m}^{3}\right)\end{array}$ & $\begin{array}{l}\mathrm{G}_{12} \\
(\mathrm{Gpa})\end{array}$ & $\begin{array}{l}\mathrm{D}_{22} \\
\left(\mathrm{~Pa} \mathrm{~m}^{3}\right)\end{array}$ & $\begin{array}{l}\mathrm{D}_{66} \\
\left(\mathrm{~Pa} \cdot \mathrm{m}^{3}\right)\end{array}$ & $\mathrm{R}=\mathrm{a} / \mathrm{b}$ & $\begin{array}{l}\mathrm{N}_{\text {th }} \\
(\mathrm{N} / \mathrm{m})\end{array}$ \\
\hline 0.20 & 5.78 & 2.96 & 0.75 & 22.81 & 10.95 & 0.98 & 20.72 & 3.49 & 2 & 1534.93 \\
\hline 0.25 & 6.78 & 3.23 & 0.75 & 26.45 & 12.62 & 1.07 & 23.80 & 3.81 & 2 & 1755.72 \\
\hline 0.30 & 7.78 & 3.52 & 0.75 & 29.96 & 14.11 & 1.17 & 26.78 & 4.17 & 2 & 1967.91 \\
\hline 0.35 & 8.78 & 3.84 & 0.75 & 33.29 & 15.41 & 1.28 & 29.62 & 4.58 & 2 & 2170.76 \\
\hline 0.40 & 9.78 & 4.20 & 0.75 & 36.44 & 16.51 & 1.41 & 32.36 & 5.04 & 2 & 2364.98 \\
\hline \multicolumn{11}{|c|}{$\psi_{\mathrm{c}}=0.2$ (constant particle ratio)- Laminated plates $7(0 / 90)$, with $\beta=1, \mathrm{~b}=0.4 \mathrm{~m}$ and $\mathrm{m}=\mathrm{n}=1$. } \\
\hline $\begin{array}{l}\Psi_{\mathrm{a}} \\
(\%)\end{array}$ & $\begin{array}{c}\mathrm{E}_{11} \\
(\mathrm{Gpa})\end{array}$ & $\begin{array}{c}\mathrm{E}_{22} \\
(\mathrm{Gpa}) \\
\end{array}$ & $\mathrm{R}_{\mathrm{e}}$ & $\begin{array}{c}\mathrm{D}_{11} \\
\left(\mathrm{~Pa} \cdot \mathrm{m}^{3}\right)\end{array}$ & $\begin{array}{c}\mathrm{D}_{12} \\
\left(\mathrm{~Pa}^{3} \mathrm{~m}^{3}\right) \\
\end{array}$ & $\begin{array}{c}\mathrm{G}_{12} \\
(\mathrm{Gpa})\end{array}$ & $\begin{array}{c}\mathrm{D}_{22} \\
\left(\mathrm{~Pa} \cdot \mathrm{m}^{3}\right) \\
\end{array}$ & $\begin{array}{c}\mathrm{D}_{66} \\
\left(\mathrm{~Pa}^{3} \mathrm{~m}^{3}\right) \\
\end{array}$ & $\mathrm{R}=\mathrm{a} / \mathrm{b}$ & $\begin{array}{l}\mathrm{N}_{\mathrm{th}} \\
(\mathrm{N} / \mathrm{m})\end{array}$ \\
\hline 0.20 & 5.78 & 2.96 & 1.33 & 24.73 & 10.95 & 0.98 & 18.79 & 3.49 & 2 & 1445.87 \\
\hline 0.25 & 6.78 & 3.23 & 1.33 & 28.90 & 12.62 & 1.07 & 21.36 & 3.81 & 2 & 1642.68 \\
\hline 0.30 & 7.78 & 3.52 & 1.33 & 32.89 & 14.11 & 1.17 & 23.85 & 4.17 & 2 & 1832.33 \\
\hline 0.35 & 8.78 & 3.84 & 1.33 & 36.66 & 15.41 & 1.28 & 26.25 & 4.58 & 2 & 2014.63 \\
\hline 0.40 & 9.78 & 4.20 & 1.33 & 40.21 & 16.51 & 1.41 & 28.59 & 5.04 & 2 & 2190.65 \\
\hline
\end{tabular}

\subsection{The buckling of the three-phase orthotropic plate under biaxial compression load}

Table 1. Effect of fiber ratio on critical force of plate under biaxial compression load (figure 3)

\begin{tabular}{|c|c|c|c|c|c|c|c|c|c|c|}
\hline $\begin{array}{l}\Psi_{\mathrm{c}} \\
(\%)\end{array}$ & $\begin{array}{l}\mathrm{E}_{11} \\
(\mathrm{Gpa})\end{array}$ & $\begin{array}{l}\mathrm{E}_{22} \\
(\mathrm{Gpa})\end{array}$ & $\mathrm{R}_{\mathrm{e}}$ & $\begin{array}{l}\mathrm{D}_{11} \\
\left(\mathrm{~Pa} \cdot \mathrm{m}^{3}\right)\end{array}$ & $\begin{array}{l}\mathrm{D}_{12} \\
\left(\mathrm{~Pa} \cdot \mathrm{m}^{3}\right)\end{array}$ & $\begin{array}{l}\mathrm{G}_{12} \\
(\mathrm{Gpa})\end{array}$ & $\begin{array}{l}\mathrm{D}_{22} \\
\left(\mathrm{~Pa} \cdot \mathrm{m}^{3}\right)\end{array}$ & $\begin{array}{l}\mathrm{D}_{66} \\
\left(\mathrm{~Pa}_{\mathrm{m}} \mathrm{m}^{3}\right)\end{array}$ & $\mathrm{R}=\mathrm{a} / \mathrm{b}$ & $\begin{array}{l}\mathrm{N}_{\mathrm{th}} \\
(\mathrm{N} / \mathrm{m})\end{array}$ \\
\hline 0.20 & 5.78 & 2.96 & 0.75 & 22.81 & 10.95 & 0.98 & 20.72 & 3.49 & 2 & 1534.93 \\
\hline 0.25 & 5.87 & 3.12 & 0.75 & 22.90 & 10.77 & 1.04 & 20.90 & 3.71 & 2 & 1551.00 \\
\hline 0.30 & 5.96 & 3.28 & 0.75 & 23.03 & 10.61 & 1.11 & 21.13 & 3.95 & 2 & 1570.60 \\
\hline 0.35 & 6.06 & 3.46 & 0.75 & 23.22 & 10.46 & 1.18 & 21.41 & 4.20 & 2 & 1593.66 \\
\hline 0.40 & 6.17 & 3.64 & 0.75 & 23.45 & 10.33 & 1.25 & 21.72 & 4.48 & 2 & 1620.19 \\
\hline \multicolumn{11}{|c|}{$\psi_{\mathrm{a}}=0.2$ (constant fiber ratio) - Laminated plates $7(0 / 90)$, with $\beta=1, b=0.4 \mathrm{~m}$ and $\mathrm{m}=\mathrm{n}=1$. } \\
\hline $\begin{array}{l}\Psi_{\mathrm{c}} \\
(\%)\end{array}$ & $\begin{array}{l}\mathrm{E}_{11} \\
(\mathrm{Gpa})\end{array}$ & $\begin{array}{l}\mathrm{E}_{22} \\
(\mathrm{Gpa})\end{array}$ & $\mathrm{R}_{\mathrm{e}}$ & $\begin{array}{l}\mathrm{D}_{11} \\
\left(\mathrm{~Pa}_{\mathrm{m}} \mathrm{m}^{3}\right)\end{array}$ & $\begin{array}{l}\mathrm{D}_{12} \\
\left(\mathrm{~Pa}_{\mathrm{m}} \mathrm{m}^{3}\right)\end{array}$ & $\begin{array}{l}\mathrm{G}_{12} \\
(\mathrm{Gpa})\end{array}$ & $\begin{array}{l}\mathrm{D}_{22} \\
\left(\mathrm{~Pa} \cdot \mathrm{m}^{3}\right)\end{array}$ & $\begin{array}{l}\mathrm{D}_{66} \\
\left(\mathrm{~Pa}_{\mathrm{m}} \mathrm{m}^{3}\right)\end{array}$ & $\mathrm{R}=\mathrm{a} / \mathrm{b}$ & $\begin{array}{l}\mathrm{N}_{\text {th }} \\
(\mathrm{N} / \mathrm{m})\end{array}$ \\
\hline 0.20 & 5.78 & 2.96 & 1.33 & 24.73 & 10.95 & 0.98 & 18.79 & 3.49 & 2 & 1445.87 \\
\hline 0.25 & 5.87 & 3.12 & 1.33 & 24.73 & 10.77 & 1.04 & 19.07 & 3.71 & 2 & 1466.01 \\
\hline 0.30 & 5.96 & 3.28 & 1.33 & 24.79 & 10.61 & 1.11 & 19.38 & 3.95 & 2 & 1489.51 \\
\hline 0.35 & 6.06 & 3.46 & 1.33 & 24.89 & 10.46 & 1.18 & 19.73 & 4.20 & 2 & 1516.34 \\
\hline 0.40 & 6.17 & 3.64 & 1.33 & 25.04 & 10.33 & 1.25 & 20.13 & 4.48 & 2 & 1546.52 \\
\hline
\end{tabular}

Table 2. Effect of particle ratio on critical force of plate under biaxial compression load (figure 4) 


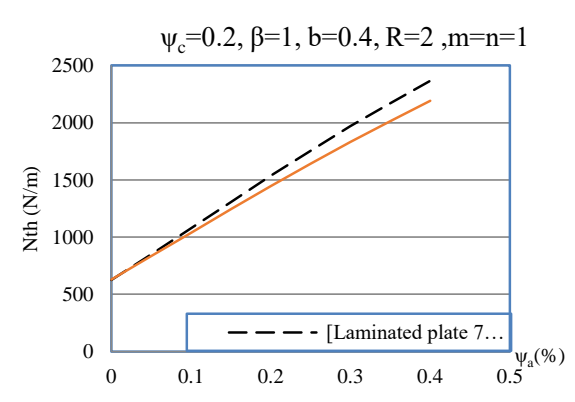

Figure 3. Effect of fiber ratio on critical force of plate under biaxial compression load.

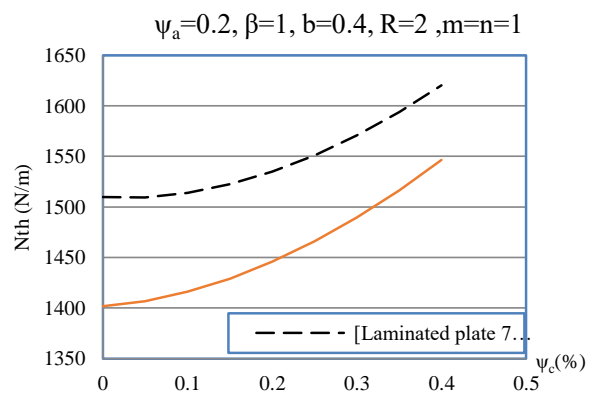

Figure 4. Effect of particle ratio on critical force of plate under biaxial compression load.

\section{Comment:}

- When the fiber and particle volume fraction ratio increase, the critical loads of the plate increase. Moreover, the effect of fiber volume fraction on the buckling of composite plate is better than one of the particle volume fraction.

- Layer placement sequence affects the buckling of plates, the value between two plates differs from $5 \div 8 \%$ (plate $7(90 / 0)$ has force-bearing capacity better than plate $7(0 / 90)$ ).

Table 3. Effect $\mathrm{R}=\mathrm{a} / \mathrm{b}$ on the critical force of plate under biaxial compression load (figure 5)

\begin{tabular}{|c|c|c|c|c|c|c|c|c|c|c|}
\hline \multicolumn{11}{|c|}{$\psi_{\mathrm{c}}=0.2$ and $\psi_{\mathrm{a}}=0.4-$ Laminated plates $7(90 / 0)$, with $\beta=1, \mathrm{~b}=0.4 \mathrm{~m}$ and $\mathrm{m}=\mathrm{n}=1$. } \\
\hline $\mathrm{R}=\mathrm{a} / \mathrm{b}$ & $\begin{array}{l}\psi_{\mathrm{a}} \\
(\%)\end{array}$ & $\begin{array}{l}E_{11} \\
(\mathrm{Gpa})\end{array}$ & $\begin{array}{l}\mathrm{E}_{22} \\
(\mathrm{Gpa})\end{array}$ & $\mathrm{R}_{\mathrm{e}}$ & $\begin{array}{l}\mathrm{D}_{11} \\
\left(\mathrm{~Pa} \cdot \mathrm{m}^{3}\right)\end{array}$ & $\begin{array}{l}\mathrm{D}_{12} \\
\left(\mathrm{~Pa} \cdot \mathrm{m}^{3}\right)\end{array}$ & $\begin{array}{l}\mathrm{G}_{12} \\
(\mathrm{Gpa})\end{array}$ & $\begin{array}{l}\mathrm{D}_{22} \\
\left(\mathrm{~Pa} \mathrm{~m}^{3}\right)\end{array}$ & $\begin{array}{l}\mathrm{D}_{66} \\
\left(\mathrm{~Pa} \cdot \mathrm{m}^{3}\right)\end{array}$ & $\begin{array}{l}\mathrm{N}_{\mathrm{th}} \\
(\mathrm{N} / \mathrm{m})\end{array}$ \\
\hline 1.00 & 0.40 & 9.78 & 4.20 & 0.75 & 36.44 & 16.51 & 1.41 & 32.36 & 5.04 & 3761.70 \\
\hline 2.00 & 0.40 & 9.78 & 4.20 & 0.75 & 36.44 & 16.51 & 1.41 & 32.36 & 5.04 & 2364.98 \\
\hline 4.00 & 0.40 & 9.78 & 4.20 & 0.75 & 36.44 & 16.51 & 1.41 & 32.36 & 5.04 & 2079.61 \\
\hline 6.00 & 0.40 & 9.78 & 4.20 & 0.75 & 36.44 & 16.51 & 1.41 & 32.36 & 5.04 & 2032.22 \\
\hline 8.00 & 0.40 & 9.78 & 4.20 & 0.75 & 36.44 & 16.51 & 1.41 & 32.36 & 5.04 & 2016.13 \\
\hline \multicolumn{11}{|c|}{$\psi_{\mathrm{c}}=0.2$ and $\psi_{\mathrm{a}}=0.4-$ Laminated plates $7(0 / 90)$, with $\beta=1, \mathrm{~b}=0.4 \mathrm{~m}$ and $\mathrm{m}=\mathrm{n}=1$. } \\
\hline $\mathrm{R}=\mathrm{a} / \mathrm{b}$ & $\begin{array}{l}\psi_{\mathrm{a}} \\
(\%)\end{array}$ & $\begin{array}{l}\mathrm{E}_{11} \\
(\mathrm{Gpa})\end{array}$ & $\begin{array}{l}\mathrm{E}_{22} \\
(\mathrm{Gpa})\end{array}$ & $\mathrm{R}_{\mathrm{e}}$ & $\begin{array}{l}\mathrm{D}_{11} \\
\left(\mathrm{~Pa} \cdot \mathrm{m}^{3}\right)\end{array}$ & $\begin{array}{l}\mathrm{D}_{12} \\
\left(\mathrm{~Pa} \mathrm{~m}^{3}\right)\end{array}$ & $\begin{array}{l}\mathrm{G}_{12} \\
(\mathrm{Gpa})\end{array}$ & $\begin{array}{l}\mathrm{D}_{22} \\
\left(\mathrm{~Pa} \cdot \mathrm{m}^{3}\right)\end{array}$ & $\begin{array}{l}\mathrm{D}_{66} \\
\left(\mathrm{~Pa}_{\mathrm{a}} \mathrm{m}^{3}\right)\end{array}$ & $\begin{array}{l}\mathrm{N}_{\mathrm{th}} \\
(\mathrm{N} / \mathrm{m})\end{array}$ \\
\hline 1.00 & 0.40 & 9.78 & 4.20 & 1.33 & 40.21 & 16.51 & 1.41 & 28.59 & 5.04 & 3761.70 \\
\hline 2.00 & 0.40 & 9.78 & 4.20 & 1.33 & 40.21 & 16.51 & 1.41 & 28.59 & 5.04 & 2190.65 \\
\hline 4.00 & 0.40 & 9.78 & 4.20 & 1.33 & 40.21 & 16.51 & 1.41 & 28.59 & 5.04 & 1861.71 \\
\hline 6.00 & 0.40 & 9.78 & 4.20 & 1.33 & 40.21 & 16.51 & 1.41 & 28.59 & 5.04 & 1806.24 \\
\hline 8.00 & 0.40 & 9.78 & 4.20 & 1.33 & 40.21 & 16.51 & 1.41 & 28.59 & 5.04 & 1787.33 \\
\hline
\end{tabular}

Table 4. Effect of thickness on critical force of plate under biaxial compression load (figure 6)

\begin{tabular}{|c|c|c|c|c|c|c|c|c|c|c|}
\hline \multicolumn{11}{|c|}{$\psi_{\mathrm{c}}=0.2$ and $\psi_{\mathrm{a}}=0.4$ - Laminated plates $5(90 / 0) \div 11(90 / 0)$, with $\beta=1, \mathrm{~m}=\mathrm{n}=1, \mathrm{~b}=0.4 \mathrm{~m}$ and $\mathrm{R}=2$. } \\
\hline $\begin{array}{l}\mathrm{E} \\
(\mathrm{m})\end{array}$ & $\begin{array}{l}E_{11} \\
(\mathrm{Gpa})\end{array}$ & $\begin{array}{l}\mathrm{E}_{22} \\
(\mathrm{Gpa})\end{array}$ & $\mathrm{R}_{\mathrm{e}}$ & $\alpha$ & $\begin{array}{l}\mathrm{D}_{11} \\
\left(\mathrm{~Pa} \cdot \mathrm{m}^{3}\right)\end{array}$ & $\begin{array}{l}\mathrm{D}_{12} \\
\left(\mathrm{~Pa} \cdot \mathrm{m}^{3}\right)\end{array}$ & $\begin{array}{l}\mathrm{G}_{12} \\
(\mathrm{Gpa})\end{array}$ & $\begin{array}{l}\mathrm{D}_{22} \\
\left(\mathrm{~Pa} \cdot \mathrm{m}^{3}\right)\end{array}$ & $\begin{array}{l}\mathrm{D}_{66} \\
\left(\mathrm{~Pa}_{\mathrm{m}} \mathrm{m}^{3}\right)\end{array}$ & $\begin{array}{l}\mathrm{N}_{\mathrm{th}} \\
(\mathrm{N} / \mathrm{m})\end{array}$ \\
\hline 0.0025 & 9.78 & 4.20 & 0.67 & 0.39 & 13.63 & 6.02 & 1.41 & 11.44 & 1.84 & 845.66 \\
\hline 0.0035 & 9.78 & 4.20 & 0.75 & 0.43 & 36.44 & 16.51 & 1.41 & 32.36 & 5.04 & 2364.98 \\
\hline 0.0045 & 9.78 & 4.20 & 0.80 & 0.44 & 76.44 & 35.08 & 1.41 & 69.78 & 10.71 & 5073.53 \\
\hline 0.0055 & 9.78 & 4.20 & 0.83 & 0.45 & 138.31 & 64.06 & 1.41 & 128.66 & 19.55 & 9321.11 \\
\hline
\end{tabular}

$\psi_{\mathrm{c}}=0.2$ and $\psi_{\mathrm{a}}=0.4$ - Laminated plates $5(0 / 90) \div 11(0 / 90)$, with $\beta=1, \mathrm{~m}=\mathrm{n}=1, \mathrm{~b}=0.4 \mathrm{~m}$, and $\mathrm{R}=2$. 


\begin{tabular}{lllllllllll}
\hline $\begin{array}{l}\mathrm{E} \\
(\mathrm{m})\end{array}$ & $\begin{array}{l}\mathrm{E}_{11} \\
(\mathrm{Gpa})\end{array}$ & $\begin{array}{l}\mathrm{E}_{22} \\
(\mathrm{Gpa})\end{array}$ & $\mathrm{R}_{\mathrm{e}}$ & $\alpha$ & $\begin{array}{l}\mathrm{D}_{11} \\
\left(\mathrm{~Pa} . \mathrm{m}^{3}\right)\end{array}$ & $\begin{array}{l}\mathrm{D}_{12} \\
\left(\mathrm{~Pa} . \mathrm{m}^{3}\right)\end{array}$ & $\begin{array}{l}\mathrm{G}_{12} \\
(\mathrm{Gpa})\end{array}$ & $\begin{array}{l}\mathrm{D}_{22} \\
\left(\mathrm{~Pa} . \mathrm{m}^{3}\right)\end{array}$ & $\begin{array}{l}\mathrm{D}_{66} \\
\left(\mathrm{~Pa} . \mathrm{m}^{3}\right)\end{array}$ & $\begin{array}{l}\mathrm{N}_{\mathrm{th}} \\
(\mathrm{N} / \mathrm{m})\end{array}$ \\
\hline 0.0025 & 9.78 & 4.20 & 1.50 & 0.21 & 15.46 & 6.02 & 1.41 & 9.61 & 1.84 & 760.96 \\
0.0035 & 9.78 & 4.20 & 1.33 & 0.29 & 40.20 & 16.51 & 1.41 & 28.60 & 5.04 & 2191.34 \\
0.0045 & 9.78 & 4.20 & 1.25 & 0.33 & 82.77 & 35.08 & 1.41 & 63.45 & 10.71 & 4780.51 \\
0.0055 & 9.78 & 4.20 & 1.20 & 0.36 & 147.96 & 64.06 & 1.41 & 119.02 & 19.55 & 8874.74 \\
\hline
\end{tabular}

\section{Comment:}

- When the $\mathrm{R}$ coefficient increases, the critical force of plate bearing two-direction compression decreases, rapidly at first then slowly to approach the smallest value $\mathrm{N}_{\mathrm{xmin}}=-\frac{\mathrm{K}_{\mathrm{x}}}{\pi^{2}} \frac{\pi^{2} \mathrm{D}_{22}}{\mathrm{~b}^{2}}=$ 1995.84 và $1763.4\left(\frac{\mathrm{N}}{\mathrm{m}}\right)$ in the order of layers $7(90 / 0)$ and $7(0 / 90)$ [since $\beta=1$, plate bearing uniform compression, according to [19] this case is hydrostatic pressure $\left(\sigma_{y} / \sigma_{x}=1\right)$ then the buckling parameter is: $\left.\frac{\mathrm{K}_{\mathrm{x}}}{\pi^{2}}=1\right]$.

- When the thickness increases, the force-bearing capacity of the plate increases, layer 7(90/0) has better force-bearing capacity than layer $7(0 / 90)$ from $5 \div 11 \%$.

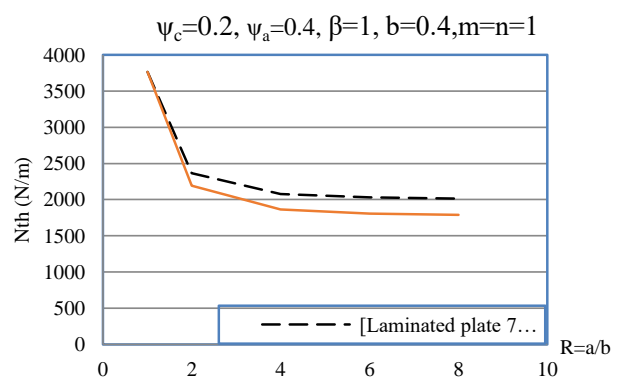

Figure 5. Effect $R=a / b$ on the critical force of plate under biaxial compression load.

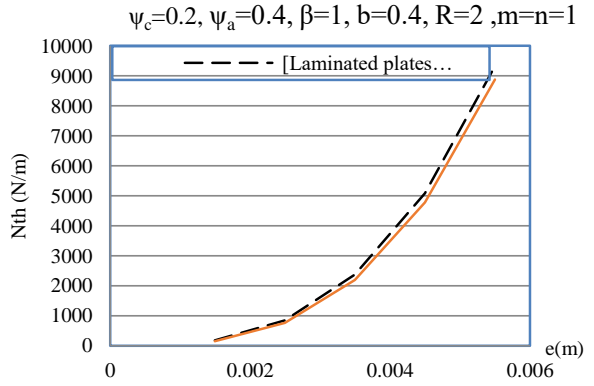

Figure 6. Effect of thickness on critical force of plate under biaxial compression load.

\subsection{The buckling of the three-phase orthotropic plate subjected to an axial compression}

Replace the values (18) into the formula (17) to have results shown in the following tables:

Table 5. Effect of fiber ratio on critical force of plate under axial compression load (figure 7).

\begin{tabular}{lllllllllll}
\hline \multicolumn{8}{l}{$\Psi_{\mathrm{c}}=0.2$ (constant particle ratio) - Laminated plates $7(90 / 0)$, with $\beta=0, \mathrm{~b}=0.4 \mathrm{~m}$ and $\mathrm{m}=\mathrm{n}=1}$. \\
\hline $\begin{array}{l}\Psi_{\mathrm{a}} \\
(\%)\end{array}$ & $\begin{array}{l}\mathrm{E}_{11} \\
(\mathrm{Gpa})\end{array}$ & $\begin{array}{l}\mathrm{E}_{22} \\
(\mathrm{Gpa})\end{array}$ & $\mathrm{R}_{\mathrm{e}}$ & $\begin{array}{l}\mathrm{D}_{11} \\
\left(\mathrm{~Pa} . \mathrm{m}^{3}\right)\end{array}$ & $\begin{array}{l}\mathrm{D}_{12} \\
\left(\mathrm{~Pa} \cdot \mathrm{m}^{3}\right)\end{array}$ & $\begin{array}{l}\mathrm{G}_{12} \\
(\mathrm{Gpa})\end{array}$ & $\begin{array}{l}\mathrm{D}_{22} \\
\left(\mathrm{~Pa} \cdot \mathrm{m}^{3}\right)\end{array}$ & $\begin{array}{l}\mathrm{D}_{66} \\
\left(\mathrm{~Pa} . \mathrm{m}^{3}\right)\end{array}$ & $\mathrm{R}=\mathrm{a} / \mathrm{b}$ & $\begin{array}{l}\mathrm{N}_{\mathrm{th}} \\
(\mathrm{N} / \mathrm{m})\end{array}$ \\
\hline 0.20 & 5.78 & 2.96 & 0.75 & 22.81 & 10.95 & 0.98 & 20.72 & 3.49 & 1.449 & 5563.17 \\
0.25 & 6.78 & 3.23 & 0.75 & 26.45 & 12.62 & 1.07 & 23.80 & 3.81 & 1.452 & 6367.34 \\
0.30 & 7.78 & 3.52 & 0.75 & 29.96 & 14.11 & 1.17 & 26.78 & 4.17 & 1.454 & 7138.42 \\
0.35 & 8.78 & 3.84 & 0.75 & 33.29 & 15.41 & 1.28 & 29.62 & 4.58 & 1.456 & 7873.51 \\
0.40 & 9.78 & 4.20 & 0.75 & 36.44 & 16.51 & 1.41 & 32.36 & 5.04 & 1.457 & 8574.99 \\
\hline
\end{tabular}




\begin{tabular}{|c|c|c|c|c|c|c|c|c|c|c|}
\hline \multicolumn{11}{|c|}{$\psi_{c}=0.2$ (constant particle ratio) - Laminated plates $7(0 / 90)$, with $\beta=0, b=0.4 m$ and $m=n=1$. } \\
\hline $\begin{array}{l}\Psi_{\mathrm{a}} \\
(\%)\end{array}$ & $\begin{array}{l}\mathrm{E}_{11} \\
\text { (Gpa) }\end{array}$ & $\begin{array}{l}\mathrm{E}_{22} \\
(\mathrm{Gpa})\end{array}$ & $\mathrm{R}_{\mathrm{e}}$ & $\begin{array}{l}\mathrm{D}_{11} \\
\left(\mathrm{~Pa} \cdot \mathrm{m}^{3}\right)\end{array}$ & $\begin{array}{l}\mathrm{D}_{12} \\
\left(\mathrm{~Pa}^{3} \mathrm{~m}^{3}\right)\end{array}$ & $\begin{array}{l}\mathrm{G}_{12} \\
(\mathrm{Gpa})\end{array}$ & $\begin{array}{l}\mathrm{D}_{22} \\
\left(\mathrm{~Pa}^{3} \mathrm{~m}^{3}\right)\end{array}$ & $\begin{array}{l}\mathrm{D}_{66} \\
\left(\mathrm{~Pa} \mathrm{~m}^{3}\right)\end{array}$ & $\mathrm{R}=\mathrm{a} / \mathrm{b}$ & $\begin{array}{l}\mathrm{N}_{\mathrm{th}} \\
(\mathrm{N} / \mathrm{m})\end{array}$ \\
\hline 0.20 & 5.78 & 2.96 & 1.33 & 24.73 & 10.95 & 0.98 & 18.79 & 3.49 & 1.515 & 5535.65 \\
\hline 0.25 & 6.78 & 3.23 & 1.33 & 28.90 & 12.62 & 1.07 & 21.36 & 3.81 & 1.525 & 6328.89 \\
\hline 0.30 & 7.78 & 3.52 & 1.33 & 32.89 & 14.11 & 1.17 & 23.85 & 4.17 & 1.533 & 7089.39 \\
\hline 0.35 & 8.78 & 3.84 & 1.33 & 36.66 & 15.41 & 1.28 & 26.25 & 4.58 & 1.537 & 7814.84 \\
\hline 0.40 & 9.78 & 4.20 & 1.33 & 40.21 & 16.51 & 1.41 & 28.59 & 5.04 & 1.540 & 8508.09 \\
\hline
\end{tabular}

Table 6. Effect of particle ratio on critical force of plate under axial compression load (figure 8).

\begin{tabular}{|c|c|c|c|c|c|c|c|c|c|c|}
\hline \multicolumn{11}{|c|}{$\Psi_{\mathrm{a}}=0.2$ (constant fiber ratio) - Laminated plates $7(90 / 0)$, with $\beta=0, \mathrm{~b}=0.4 \mathrm{~m}$ and $\mathrm{m}=\mathrm{n}=1$. } \\
\hline $\begin{array}{l}\Psi_{\mathrm{c}} \\
(\%)\end{array}$ & $\begin{array}{l}\mathrm{E}_{11} \\
\text { (Gpa) }\end{array}$ & $\begin{array}{l}\mathrm{E}_{22} \\
(\mathrm{Gpa})\end{array}$ & $\mathrm{R}_{\mathrm{e}}$ & $\begin{array}{l}\mathrm{D}_{11} \\
\left(\mathrm{~Pa} \cdot \mathrm{m}^{3}\right)\end{array}$ & $\begin{array}{l}\mathrm{D}_{12} \\
\left(\mathrm{~Pa}^{3} \mathrm{~m}^{3}\right)\end{array}$ & $\begin{array}{l}\mathrm{G}_{12} \\
(\mathrm{Gpa})\end{array}$ & $\begin{array}{l}\mathrm{D}_{22} \\
\left(\mathrm{~Pa}^{3} \mathrm{~m}^{3}\right)\end{array}$ & $\begin{array}{l}\mathrm{D}_{66} \\
\left(\mathrm{~Pa}_{\mathrm{m}}^{3}\right)\end{array}$ & $\mathrm{R}=\mathrm{a} / \mathrm{b}$ & $\begin{array}{l}\mathrm{N}_{\mathrm{th}} \\
(\mathrm{N} / \mathrm{m})\end{array}$ \\
\hline 0.20 & 5.78 & 2.96 & 0.75 & 22.81 & 10.95 & 0.98 & 20.72 & 3.49 & 1.449 & 5563.17 \\
\hline 0.25 & 5.87 & 3.12 & 0.75 & 22.90 & 10.77 & 1.04 & 20.90 & 3.71 & 1.447 & 5618.08 \\
\hline 0.30 & 5.96 & 3.28 & 0.75 & 23.03 & 10.61 & 1.11 & 21.13 & 3.95 & 1.445 & 5685.96 \\
\hline 0.35 & 6.06 & 3.46 & 0.75 & 23.22 & 10.46 & 1.18 & 21.41 & 4.20 & 1.443 & 5766.60 \\
\hline 0.40 & 6.17 & 3.64 & 0.75 & 23.45 & 10.33 & 1.25 & 21.72 & 4.48 & 1.442 & 5859.95 \\
\hline \multicolumn{11}{|c|}{$\psi_{\mathrm{a}}=0.2$ (constant fiber ratio) - Laminated plates $7(0 / 90)$, with $\beta=0, \mathrm{~b}=0.4 \mathrm{~m}$ and $\mathrm{m}=\mathrm{n}=1$. } \\
\hline $\begin{array}{l}\Psi_{\mathrm{c}} \\
(\%)\end{array}$ & $\begin{array}{l}\mathrm{E}_{11} \\
(\mathrm{Gpa})\end{array}$ & $\begin{array}{l}\mathrm{E}_{22} \\
(\mathrm{GPa})\end{array}$ & $\mathrm{R}_{\mathrm{e}}$ & $\begin{array}{l}\mathrm{D}_{11} \\
\left(\mathrm{~Pa}_{\mathrm{m}} \mathrm{m}^{3}\right)\end{array}$ & $\begin{array}{l}\mathrm{D}_{12} \\
\left(\mathrm{~Pa} \cdot \mathrm{m}^{3}\right)\end{array}$ & $\begin{array}{l}\mathrm{G}_{12} \\
(\mathrm{GPa})\end{array}$ & $\begin{array}{l}\mathrm{D}_{22} \\
\left(\mathrm{~Pa} \cdot \mathrm{m}^{3}\right)\end{array}$ & $\begin{array}{l}\mathrm{D}_{66} \\
\left(\mathrm{~Pa} \mathrm{~m}^{3}\right)\end{array}$ & $\mathrm{R}=\mathrm{a} / \mathrm{b}$ & $\begin{array}{l}\mathrm{N}_{\mathrm{th}} \\
(\mathrm{N} / \mathrm{m})\end{array}$ \\
\hline 0.20 & 5.78 & 2.96 & 1.33 & 24.73 & 10.95 & 0.98 & 18.79 & 3.49 & 1.515 & 5535.65 \\
\hline 0.25 & 5.87 & 3.12 & 1.33 & 24.73 & 10.77 & 1.04 & 19.07 & 3.71 & 1.509 & 5593.19 \\
\hline 0.30 & 5.96 & 3.28 & 1.33 & 24.79 & 10.61 & 1.11 & 19.38 & 3.95 & 1.504 & 5663.50 \\
\hline 0.35 & 6.06 & 3.46 & 1.33 & 24.89 & 10.46 & 1.18 & 19.73 & 4.20 & 1.499 & 5746.39 \\
\hline 0.40 & 6.17 & 3.64 & 1.33 & 25.04 & 10.33 & 1.25 & 20.13 & 4.48 & 1.494 & 5841.84 \\
\hline
\end{tabular}

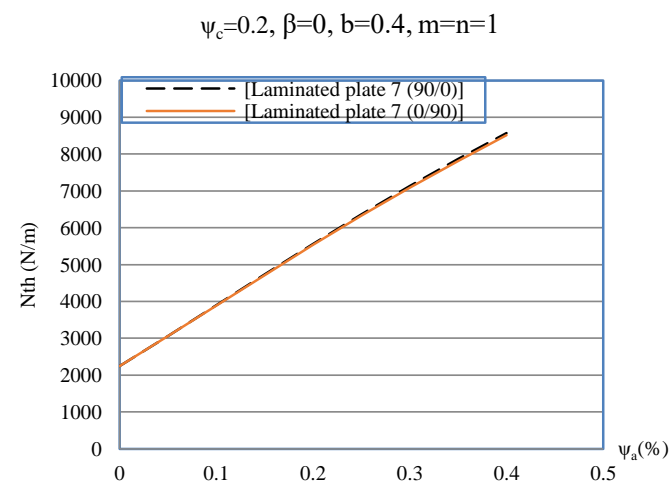

Figure 7. Effect of fiber ratio on critical force of plate under axial compression load.

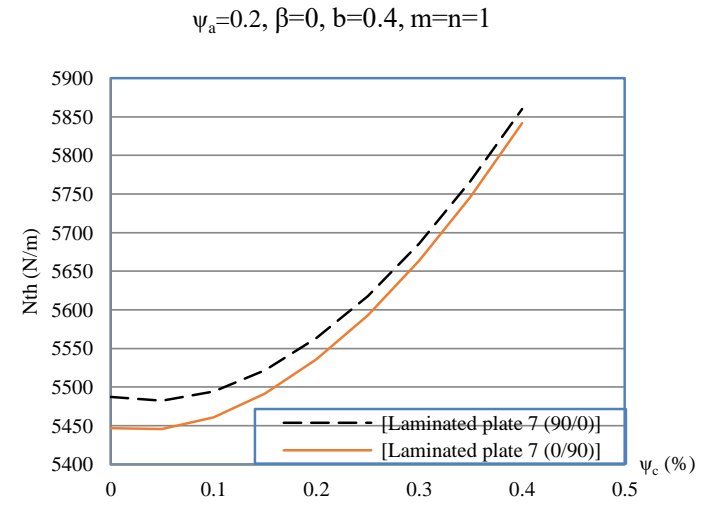

Figure 8. Effect of particle ratio on critical force of plate under axial compression load. 


\section{Comment:}

- When the fiber and particle volume fraction ratio increase the one-direction compression resistance of the plate increase, the effect of fiber on the plate's buckling is better than the particle.

- Plates of the same size have force-bearing capacity in one direction at least 3.6 times better than in two directions.

Table 7. Effect $R=a / b$ on the critical force of plate under axial compression load (figure 9 )

\begin{tabular}{|c|c|c|c|c|c|c|c|c|c|c|}
\hline $\mathrm{R}=\mathrm{a} / \mathrm{b}$ & $\begin{array}{l}\psi_{\mathrm{a}} \\
(\%)\end{array}$ & $\begin{array}{l}\mathrm{E}_{11} \\
(\mathrm{GPa})\end{array}$ & $\begin{array}{l}E_{22} \\
(\mathrm{GPa})\end{array}$ & $\mathrm{R}_{\mathrm{e}}$ & $\begin{array}{l}\mathrm{D}_{11} \\
\left(\mathrm{~Pa} \cdot \mathrm{m}^{3}\right)\end{array}$ & $\begin{array}{l}\mathrm{D}_{12} \\
\left(\mathrm{~Pa} \cdot \mathrm{m}^{3}\right)\end{array}$ & $\begin{array}{l}\mathrm{G}_{12} \\
(\mathrm{GPa})\end{array}$ & $\begin{array}{l}\mathrm{D}_{22} \\
\left(\mathrm{~Pa} \cdot \mathrm{m}^{3}\right)\end{array}$ & $\begin{array}{l}\mathrm{D}_{66} \\
\left(\mathrm{~Pa} \cdot \mathrm{m}^{3}\right)\end{array}$ & $\begin{array}{l}\mathrm{N}_{\text {th }} \\
(\mathrm{N} / \mathrm{m})\end{array}$ \\
\hline 1.457 & 0.40 & 9.78 & 4.20 & 0.75 & 36.44 & 16.51 & 1.41 & 32.36 & 5.04 & 8574.99 \\
\hline 2.523 & 0.40 & 9.78 & 4.20 & 0.75 & 36.44 & 16.51 & 1.41 & 32.36 & 5.04 & 7868.93 \\
\hline 3.569 & 0.40 & 9.78 & 4.20 & 0.75 & 36.44 & 16.51 & 1.41 & 32.36 & 5.04 & 7692.41 \\
\hline 4.607 & 0.40 & 9.78 & 4.20 & 0.75 & 36.44 & 16.51 & 1.41 & 32.36 & 5.04 & 7621.81 \\
\hline 5.643 & 0.40 & 9.78 & 4.20 & 0.75 & 36.44 & 16.51 & 1.41 & 32.36 & 5.04 & 7586.50 \\
\hline \multicolumn{11}{|c|}{$\psi_{\mathrm{c}}=0.2$ - Laminated plates $7(0 / 90)$, with $\beta=0, \mathrm{~b}=0.4 \mathrm{~m}$ and $\mathrm{m}=1 \div 5, \mathrm{n}=1$. } \\
\hline $\mathrm{R}=\mathrm{a} / \mathrm{b}$ & $\begin{array}{l}\psi_{\mathrm{a}} \\
(\%)\end{array}$ & $\begin{array}{l}\mathrm{E}_{11} \\
\text { (GPa) }\end{array}$ & $\begin{array}{l}\mathrm{E}_{22} \\
(\mathrm{GPa})\end{array}$ & $\mathrm{R}_{\mathrm{e}}$ & $\begin{array}{l}\mathrm{D}_{11} \\
\left(\mathrm{~Pa}^{3} \mathrm{~m}^{3}\right)\end{array}$ & $\begin{array}{l}\mathrm{D}_{12} \\
\left(\mathrm{~Pa} \cdot \mathrm{m}^{3}\right)\end{array}$ & $\begin{array}{l}\mathrm{G}_{12} \\
(\mathrm{GPa})\end{array}$ & $\begin{array}{l}\mathrm{D}_{22} \\
\left(\text { Pa.m }^{3}\right)\end{array}$ & $\begin{array}{l}\mathrm{D}_{66} \\
\left(\mathrm{~Pa} \cdot \mathrm{m}^{3}\right)\end{array}$ & $\begin{array}{l}\mathrm{N}_{\text {th }} \\
(\mathrm{N} / \mathrm{m})\end{array}$ \\
\hline 1.540 & 0.40 & 9.78 & 4.20 & 1.33 & 40.21 & 16.51 & 1.41 & 28.59 & 5.04 & 8508.09 \\
\hline 2.668 & 0.40 & 9.78 & 4.20 & 1.33 & 40.21 & 16.51 & 1.41 & 28.59 & 5.04 & 7810.95 \\
\hline 3.773 & 0.40 & 9.78 & 4.20 & 1.33 & 40.21 & 16.51 & 1.41 & 28.59 & 5.04 & 7636.66 \\
\hline 4.870 & 0.40 & 9.78 & 4.20 & 1.33 & 40.21 & 16.51 & 1.41 & 28.59 & 5.04 & 7566.95 \\
\hline 5.965 & 0.40 & 9.78 & 4.20 & 1.33 & 40.21 & 16.51 & 1.41 & 28.59 & 5.04 & 7532.09 \\
\hline
\end{tabular}

Table 8. Effect of thickness on critical force of plate under axial compression load (figure 10)

\begin{tabular}{|c|c|c|c|c|c|c|c|c|c|c|}
\hline \multicolumn{11}{|c|}{$\psi_{\mathrm{c}}=0.2$ and $\psi_{\mathrm{a}}=0.4-$ Laminated plates $5(90 / 0) \div 11(90 / 0)$, with $\beta=0, \mathrm{~b}=0.4 \mathrm{~m}, \mathrm{~m}=\mathrm{n}=1$. } \\
\hline $\begin{array}{l}\mathrm{e} \\
\text { (m) }\end{array}$ & $\begin{array}{l}E_{11} \\
(\mathrm{GPa})\end{array}$ & $\begin{array}{l}\mathrm{E}_{22} \\
(\mathrm{GPa})\end{array}$ & $\mathrm{R}_{\mathrm{e}}$ & $\alpha$ & $\begin{array}{l}\mathrm{D}_{11} \\
\left(\text { Pa.m }{ }^{3}\right)\end{array}$ & $\begin{array}{l}\mathrm{D}_{12} \\
\left(\mathrm{~Pa} \cdot \mathrm{m}^{3}\right)\end{array}$ & $\begin{array}{l}\mathrm{G}_{12} \\
(\mathrm{GPa})\end{array}$ & $\begin{array}{l}\mathrm{D}_{22} \\
\left(\mathrm{~Pa}^{3} \mathrm{~m}^{3}\right)\end{array}$ & $\begin{array}{l}\mathrm{D}_{66} \\
\left(\mathrm{~Pa}^{3} \mathrm{~m}^{3}\right)\end{array}$ & $\begin{array}{l}\mathrm{N}_{\mathrm{th}} \\
(\mathrm{N} / \mathrm{m})\end{array}$ \\
\hline 0.0025 & 9.78 & 4.20 & 0.67 & 0.39 & 13.63 & 6.02 & 1.41 & 11.44 & 1.84 & 3121.02 \\
\hline 0.0035 & 9.78 & 4.20 & 0.75 & 0.43 & 36.44 & 16.51 & 1.41 & 32.36 & 5.04 & 8574.99 \\
\hline 0.0045 & 9.78 & 4.20 & 0.80 & 0.44 & 76.44 & 35.08 & 1.41 & 69.78 & 10.71 & 18233.23 \\
\hline 0.0055 & 9.78 & 4.20 & 0.83 & 0.45 & 138.31 & 64.06 & 1.41 & 128.66 & 19.55 & 33297.92 \\
\hline
\end{tabular}

\begin{tabular}{lllllllllll}
\hline \multicolumn{2}{l}{$\psi_{\mathrm{c}}=0.2$ and $\psi_{\mathrm{a}}=0.4$} & - Laminated plates $5(0 / 90) \div 11(0 / 90)$, with $\beta=0, \mathrm{~b}=0.4 \mathrm{~m}, \mathrm{~m}=\mathrm{n}=1$. \\
\hline $\begin{array}{l}\mathrm{e} \\
(\mathrm{m})\end{array}$ & $\begin{array}{l}\mathrm{E}_{11} \\
(\mathrm{GPa})\end{array}$ & $\begin{array}{l}\mathrm{E}_{22} \\
(\mathrm{GPa})\end{array}$ & $\mathrm{R}_{\mathrm{e}}$ & $\alpha$ & $\begin{array}{l}\mathrm{D}_{11} \\
\left(\mathrm{~Pa} \cdot \mathrm{m}^{3}\right)\end{array}$ & $\begin{array}{l}\mathrm{D}_{12} \\
\left(\mathrm{~Pa} . \mathrm{m}^{3}\right)\end{array}$ & $\begin{array}{l}\mathrm{G}_{12} \\
(\mathrm{GPa})\end{array}$ & $\begin{array}{l}\mathrm{D}_{22} \\
\left(\mathrm{~Pa} . \mathrm{m}^{3}\right)\end{array}$ & $\begin{array}{l}\mathrm{D}_{66} \\
\left(\mathrm{~Pa} \cdot \mathrm{m}^{3}\right)\end{array}$ & $\begin{array}{l}\mathrm{N}_{\mathrm{th}} \\
(\mathrm{N} / \mathrm{m})\end{array}$ \\
\hline 0.0025 & 9.78 & 4.20 & 1.50 & 0.21 & 15.46 & 6.02 & 1.41 & 9.61 & 1.84 & 3075.01 \\
0.0035 & 9.78 & 4.20 & 1.33 & 0.29 & 40.20 & 16.51 & 1.41 & 28.60 & 5.04 & 8508.49 \\
0.0045 & 9.78 & 4.20 & 1.25 & 0.33 & 82.77 & 35.08 & 1.41 & 63.45 & 10.71 & 18146.05 \\
0.0055 & 9.78 & 4.20 & 1.20 & 0.36 & 147.96 & 64.06 & 1.41 & 119.02 & 19.55 & 33190.04 \\
\hline
\end{tabular}




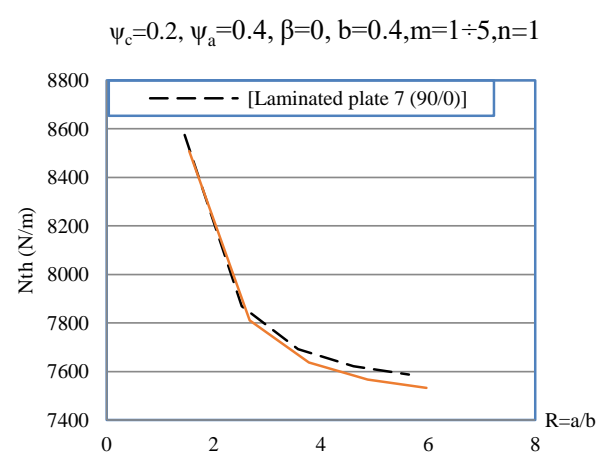

Figure 9 . Effect $R=a / b$ on the critical force of plate under axial compression load.

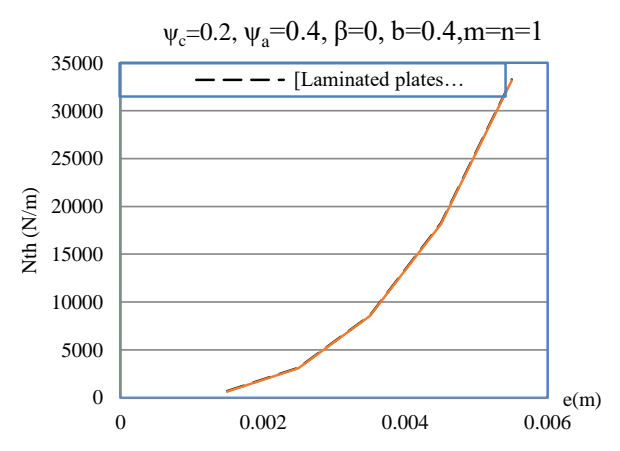

Figure 10. Effect of thickness on critical force of plate under axial compression load.

\section{Comment:}

- When the $\mathrm{R}$ coefficient increases, the critical force of the plate bearing one-direction compression decreases, first decreases, rapidly at first then slowly to approach the smallest value $\mathrm{N}_{\mathrm{xmin}}=-\frac{\mathrm{K}_{\mathrm{x}}}{\pi^{2}} \frac{\pi^{2} \mathrm{D}_{22}}{\mathrm{~b}^{2}}=7515.89$ và $7462.38\left(\frac{\mathrm{N}}{\mathrm{m}}\right)$ in the order of layers $7(90 / 0)$ and $7(0 / 90)$ [where the smallest value of buckling parameter is: $\frac{K_{x}}{\pi^{2}}=2\left(\sqrt{\frac{D_{11}}{D_{22}}}+\frac{D_{12}+2 D_{66}}{D_{22}}\right)[19]$

- When the thickness increases, the force-bearing capacity of the plate increases, layers $7(90 / 0)$ and 7(0/90) has a critical compressed force in an equivalent direction.

\section{Conclusion}

The article introduces study on the buckling of orthotropic three-phase composite plates subjected to simultaneous biaxial and axial compression load. Some conclusions are obtained:

- Static stability of the three-phase composite plate is significantly influenced by elements of material composition, particles and fibers volume fraction ratio, plate size and thickness:

When the fiber ratio increases, the compressive bearing capacity of plates strongly increases; however, when the percentage of particle increases, the compressive bearing capacity of plates less increases.

Therefore, the effect of fiber on plate buckling is much better than that of particle.

When the R-shape parameter increases, the critical force of plates subjected to simultaneous biaxial and axial compression reduces, rapidly at first then slowly to approach the smallest value (for biaxial compression $\mathrm{N}_{\mathrm{x} \text { min }}=46 \% \mathrm{Nth}(1,1)$ and axial compression $\left.\mathrm{N}_{\mathrm{x} \text { min }}=87 \% \mathrm{Nth}(\mathrm{m}, 1)\right)$. Therefore, it is necessary to select this parameter reasonably to ensure the buckling of the plate without increasing its weight.

Plates of the same size have force-bearing capacity in one direction at least 3.6 times better than in two directions.

- Layer placement sequence affects the buckling of plates, the value between two plates differs from $5 \div 11 \%$ (plate $7(90 / 0)$ has force-bearing capacity better than plate $7(0 / 90)$ ), which means that 
in terms of layer inlay: the bigger the number of layers inlaid in perpendicular direction (horizontal direction of the plate) is, the better the stability will be.

- When the thickness increases, compression and shear resistance of the plate increases.

- When the thickness increases, the force-bearing capacity of the plate increases, layer 7(90/0) has better force-bearing capacity than layer 7(0/90) from $5 \div 11 \%$.

Thus, upon adding reinforced particles to improve the criteria: increasing waterproofing, fireretardancy and the surface hardness of the plate will effect on tensile, bending, impact strength and the buckling of the plate. The aforementioned research results are the scientific basis for shipbuilding facilities to design and manufacture ship structures and equipment on board to meet the criteria: better stability, waterproofing, fire-retardancy materials with most reasonable prime price.

\section{Acknowledgements}

This research is funded by Vietnam National Foundation for Science and Technology Development (NAFOSTED) under grant number 107.02-2018.04. The author is grateful for this support.

\section{References}

[1] J.-M. Bertholot. Composite Materials. Mechanical Behavior and Structural, 1999.

[2] S. Selvaraju, S. Ilaiyavel. Application of composites in marine industry. Jers, Vol.II, Issue II, 2011, 89-91.

[3] A.N. Lagarkov, L.V. Panina, and A.K. Sarychev. Effective magnetic permeability of composite materials near the percolation threshold. J. Zh. Eksp. Teor. Fiz 1987; 93, 215-221.

[4] L. Wu, S. Pan. Bounds on effective magnetic permeability of three-phase composites with coated spherical inclusions. Compos Sci Tech 2012; 72(12):1443-50.

[5] M. Bar, R. Alagirusamy, and A. Das. Flame retardant polymer composites. J. Fibers and polymers 2015, Vol.16, No.4, 705-717

[6] N.D. Duc, T.Q. Quan, D. Nam. Nonlinear stability analysis of imperfect three phase polymer composite plates. J. Mechanics of Composite Materials 2013, Vol.49, N4, p. 345-358.

[7] N.D. Duc, P.V. Thu. Nonlinear stability analysis of imperfect three-phase polymer composite plates in thermal environments. J. Composite Structure 2014, Vol.109, pp:130-138.

[8] M.H. Shen, F.M. Chen, S.Y. Hung. Piezoelectric study for a three-phase composite containing arbitrary inclusion. Int J MechSci 2010;52(4):561-71.

[9] R.F. Rango, L.G. Nallim, S. Oller. Formulation of enriched macro elements using trigonometric shear deformation theory for free vibration analysis of symmetric laminated composite plate assemblies. Compos. Struct 2015; 119:38-49.

[10] R. Sahoo, B.N. Singh. A new trigonometric zigzag theory for buckling and free vibration analysis of laminated composite and sandwich plates. Compos Struct 2014; 117:316-32.

[11] M. Samadpour, M. Sadighi, M. Shakeri, H.A. Zamani. Vibration analysis of thermally buckled SMA hybrid composite sandwich plate. Compos Struct 2015; 119:251-63.

[12] Y. Heydarpour, M.M. Aghdam,P. Malekzadeh. Free vibration analysis of rotating functionally graded carbon nanotube-reinforced composite truncated conical shells. Compos Struct 2014; 117:187-200. 
[13] R. Burgueno, N. Hu, A. Heeringa, N. Lajnef. Tailoring the elastic postbuckling response of thin-walled cylindrical composite shells under axial compression. Thin-Wall Struct 2014;84:14-25.

[14] P.V. Thu, N.D. Duc. Nonlinear dynamic response and vibration of an imperfect three-phase laminated nanocomposite cylindrical panel resting on elastic foundations in thermal environments. J. Science and Engineering of Composite Materials 2016, DOI: 10.1515/secm-2015-046

[15] N.D. Duc, H. Hadavinia, P.V. Thu, T.Q. Quan. Vibration and nonlinear dynamic response of imperfect threephase polymer nanocomposite panel resting on elastic foundations under hydrodynamic loads. J. Composite Structure 2015, Vol.131, pp.229-237.

[16] P.V. Thu, N.D .Duc. Nonlinear stability analysis of imperfect three-phase sandwich laminated polymer nanocomposite panels resting on elastic foundations in thermal environments. Journal of Science, MathematicsPhysics 2016, Vietnam National University, Hanoi, Vol.32, N1, pp 20-36.

[17] G.A. Vanin, N.D. Duc. The theory of spherofibrous composite.1: The input relations, hypothesis and models. Mech Compos Mater 1996;32(3):291-305.

[18] G.A. Vanin. Micro-Mechanics of composite materials, "NaukaDumka", Kiev; 1985.

[19] W. Arthur Leissa. Buckling of laminated composite plates and shell panels, 1985.

[20] A.S. Volmir. Non-linear dynamics of plates and shells. Science Edition; 1972. 


\section{Appendix A:}

To determine the response of plates, laminated composite plate are placed in the coordinate system of $\mathrm{x}, \mathrm{y}, \mathrm{z}$. Where: $\mathrm{xy}$ is the middle surface of the plate and $\mathrm{z}$ is the direction according to the thickness $\mathrm{h}$ of the plate. According to Kirchhoff assumption, the deformation of the normal line with middle surface is a straight line perpendicular to the deformation surface of the middle surface. Therefore, the response of plates is represented by the following relation:

$$
u=u_{0}-z \frac{\partial w}{\partial x} ;(\text { Leissa, 1985, [19]) }
$$

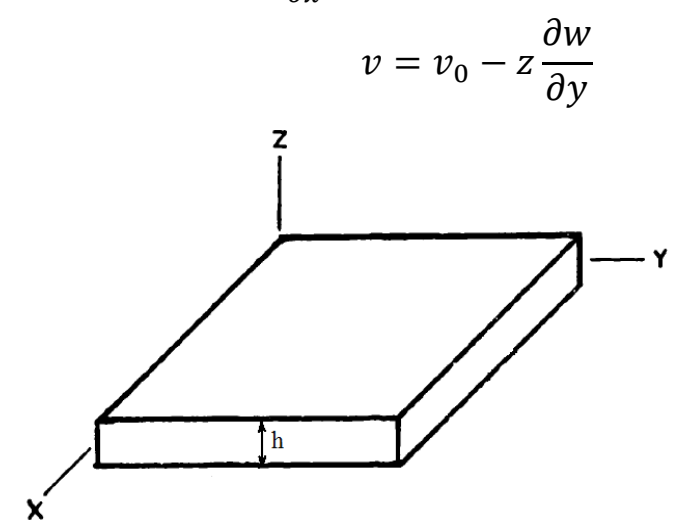

Figure 11: Laminated composite plate

Where: $\mathrm{u}, \mathrm{v}, \mathrm{w}$ are displacement components along the $\mathrm{x}, \mathrm{y}$ and $\mathrm{z}$ directions, and $\mathrm{u}_{0}, \mathrm{v}_{0}$ are displacement at one point of the middle surface. $\mathrm{u}_{0}, \mathrm{v}_{0}$ and $\mathrm{w}_{0}$ are functions of $\mathrm{x}$ and $\mathrm{y}$. Deformation displacement equations are used according to classical elasticity theory to have $[19,20]$ :

$$
\varepsilon_{x}=\frac{\partial u}{\partial x}, \quad \varepsilon_{y}=\frac{\partial v}{\partial y}, \quad \gamma_{x y}=\frac{\partial v}{\partial x}+\frac{\partial u}{\partial y}
$$

Where: $\varepsilon_{\mathrm{x}}, \varepsilon_{\mathrm{y}}$ is the deformation in $\mathrm{x}$ and $\mathrm{y}$ directions; $\gamma_{\mathrm{xy}}$ is shearing deformation, the equation (1) is rewritten as follows:

$$
\left(\begin{array}{c}
\varepsilon_{x} \\
\varepsilon_{y} \\
\gamma_{x y}
\end{array}\right)=\left(\begin{array}{c}
\varepsilon_{x}^{0} \\
\varepsilon_{y}^{0} \\
\gamma_{x y}^{0}
\end{array}\right)+z\left(\begin{array}{l}
k_{x} \\
k_{y} \\
k_{x y}
\end{array}\right),
$$

Where: $\varepsilon_{\mathrm{x}}^{0}, \varepsilon_{\mathrm{y}}^{0}$ và $\gamma_{\mathrm{xy}}^{0}$ is the deformation of middle surface, and $\mathrm{k}_{\mathrm{x}}, \mathrm{k}_{\mathrm{y}}, \mathrm{k}_{\mathrm{xy}}$ are the curvature of the plate bearing bending force.

$$
\left(\begin{array}{c}
\varepsilon_{x}^{0} \\
\varepsilon_{y}^{0} \\
\gamma_{x y}^{0}
\end{array}\right)=\left[\begin{array}{c}
\frac{\partial u_{0}}{\partial x} \\
\frac{\partial v_{0}}{\partial y} \\
\frac{\partial v_{0}}{\partial x}+\frac{\partial u_{0}}{\partial y}
\end{array}\right],\left[\begin{array}{c}
k_{x} \\
k_{y} \\
k_{x y}
\end{array}\right]=\left[\begin{array}{c}
-\frac{\partial^{2} w_{0}}{\partial x^{2}} \\
-\frac{\partial^{2} w_{0}}{\partial y^{2}} \\
-2 \frac{\partial^{2} w_{0}}{\partial x \partial y}
\end{array}\right]
$$

Hooke's law for composite plates is defined as follows: 


$$
\left(\begin{array}{l}
\sigma_{x} \\
\sigma_{y} \\
\sigma_{x y}
\end{array}\right)_{k}=\left(\begin{array}{lll}
Q_{11}^{\prime} & Q_{12}^{\prime} & Q_{16}^{\prime} \\
Q_{12}^{\prime} & Q_{22}^{\prime} & Q_{26}^{\prime} \\
Q_{16}^{\prime} & Q_{26}^{\prime} & Q_{66}^{\prime}
\end{array}\right)_{k}\left(\begin{array}{l}
\varepsilon_{x} \\
\varepsilon_{y} \\
\gamma_{x y}
\end{array}\right)_{k},
$$

Where: $\mathrm{k}$ is the number of layers

$$
\begin{aligned}
& Q_{11}^{\prime}=Q_{11} c^{4}+Q_{22} s^{4}+2\left(Q_{12}+2 Q_{66}\right) s^{2} c^{2} \\
& Q_{12}^{\prime}=\left(Q_{11}+Q_{22}-4 Q_{66}\right) c^{2} s^{2}+Q_{12}\left(s^{4}+c^{4}\right) \\
& Q_{22}^{\prime}=Q_{11} s^{4}+Q_{22} c^{4}+2\left(Q_{12}+2 Q_{66}\right) s^{2} c^{2} \\
& Q_{16}^{\prime}=\left(Q_{11}-Q_{12}-2 Q_{66}\right) c^{3} s+\left(Q_{22}-Q_{12}-2 Q_{66}\right) c s^{3} \\
& Q_{26}^{\prime}=\left(Q_{11}-Q_{12}-2 Q_{66}\right) c s^{3}+\left(Q_{22}-Q_{12}-2 Q_{66}\right) c^{3} s \\
& Q_{66}^{\prime}=\left(Q_{11}+Q_{22}-2 Q_{12}-2 Q_{66}\right) c^{2} s^{2}+Q_{66}\left(c^{4}+s^{4}\right)
\end{aligned}
$$

$s=\sin \theta ; c=\cos \theta ; \theta$ is the angle between the direction of fiber and coordinates.

And

$$
\begin{gathered}
Q_{11}=\frac{E_{11}}{1-\frac{E_{22}}{E_{11}} v_{12}^{2}}=\frac{E_{11}}{1-v_{12} v_{21}} ; \\
Q_{22}=\frac{E_{22}}{1-\frac{E_{22}}{E_{11}} v_{12}^{2}}=\frac{E_{22}}{E_{11}} Q_{11} \\
Q_{12}=\frac{v_{12} E_{22}}{1-v_{12} v_{21}}=v_{12} Q_{22} ; \quad Q_{66}=G_{12}
\end{gathered}
$$

(Where: 1,2 are direction of fiber)

Force and modulus of a composite plate are determined as follows:

$$
\begin{aligned}
& {\left[\begin{array}{l}
N_{x} \\
N_{y} \\
N_{x y}
\end{array}\right]=\int_{-h / 2}^{h / 2}\left[\begin{array}{l}
\sigma_{x x} \\
\sigma_{y y} \\
\tau_{x y}
\end{array}\right]_{k} d z} \\
& {\left[\begin{array}{l}
M_{x} \\
M_{y} \\
M_{x y}
\end{array}\right]=\int_{-h / 2}^{h / 2} z\left[\begin{array}{l}
\sigma_{x x} \\
\sigma_{y y} \\
\tau_{x y}
\end{array}\right]_{k} d z}
\end{aligned}
$$

Where: $\sigma_{\mathrm{xx}}$ and $\sigma_{\mathrm{yy}}$ are normal stresses, and $\tau_{\mathrm{xy}}$ is shear stress.

Force and modulus relationship between the middle-surface deformation and curvature of the plate is as follows: 


$$
\left[\begin{array}{c}
N_{x} \\
N_{y} \\
N_{x y} \\
M_{x} \\
M_{y} \\
M_{x y}
\end{array}\right]=\left[\begin{array}{c}
A_{11} A_{12} A_{16} B_{11} B_{12} B_{16} \\
A_{12} A_{22} A_{26} B_{12} B_{22} B_{26} \\
A_{16} A_{26} A_{66} B_{16} B_{26} B_{66} \\
B_{11} B_{12} B_{16} D_{11} D_{12} D_{16} \\
B_{12} B_{22} B_{26} D_{12} D_{22} D_{26} \\
B_{16} B_{26} B_{66} D_{16} D_{26} D_{66}
\end{array}\right]\left[\begin{array}{c}
\varepsilon_{x}^{0} \\
\varepsilon_{y}^{0} \\
\gamma_{x y}^{0} \\
k_{x} \\
k_{y} \\
k_{x y}
\end{array}\right]
$$

Or in shortened form:

$$
\left[\begin{array}{l}
N \\
M
\end{array}\right]=\left[\begin{array}{l}
A B \\
B D
\end{array}\right]\left[\begin{array}{l}
\varepsilon \\
k
\end{array}\right]
$$

Where:

$$
\begin{aligned}
& A_{i j}=\sum_{k=1}^{n}\left(Q_{\mathrm{ij}}^{\prime}\right)_{k}\left(h_{k}-h_{k-1}\right), i, j=1,2,6 \\
& B_{i j}=\frac{1}{2} \sum_{k=1}^{n}\left(Q_{\mathrm{ij}}^{\prime}\right)_{k}\left(h_{k}^{2}-h_{k-1}^{2}\right), \quad i, j=1,2,6 \\
& D_{i j}=\frac{1}{3} \sum_{k=1}^{n}\left(Q_{\mathrm{ij}}^{\prime}\right)_{k}\left(h_{k}^{3}-h_{k-1}^{3}\right), \quad i, j=1,2,6
\end{aligned}
$$

On the other hand, the plate bearing q pressure in $\mathrm{z}$ direction and membrane force $\left(\mathrm{N}_{\mathrm{x}}, \mathrm{N}_{\mathrm{y}}, \mathrm{N}_{\mathrm{xy}}\right)$. Therefore, there is an equilibrium simultaneous equation like (Leissa, 1985, [19]):

$$
\begin{gathered}
\frac{\partial N_{x}}{\partial x}+\frac{\partial N_{x y}}{\partial y}-Q_{x} \frac{\partial w_{0}}{\partial x}=0 \\
\frac{\partial N_{x y}}{\partial x}+\frac{\partial N_{y}}{\partial y}-Q_{y} \frac{\partial w_{0}}{\partial y}=0 \\
\frac{\partial Q_{x}}{\partial x}+\frac{\partial Q_{y}}{\partial y}+N_{x} \frac{\partial^{2} w_{o}}{\partial x^{2}}+2 N_{x y} \frac{\partial^{2} w_{0}}{\partial x \partial y}+N_{y} \frac{\partial^{2} w_{0}}{\partial y^{2}}+q=0
\end{gathered}
$$

And:

$$
\begin{aligned}
& Q_{x}=\frac{\partial M_{x}}{\partial x}+\frac{\partial M_{x y}}{\partial y} \\
& Q_{y}=\frac{\partial M_{x y}}{\partial x}+\frac{\partial M_{y}}{\partial y}
\end{aligned}
$$

The transverse shear forces $\mathrm{Q}_{\mathrm{x}}$ and $\mathrm{Q}_{\mathrm{y}}$, as well as the slopes $\frac{\partial \mathrm{w}_{0}}{\partial \mathrm{x}}$ and $\frac{\partial \mathrm{w}_{0}}{\partial \mathrm{y}}$ are typically small in the two of equations (13a) and (13b). Taking advantage of this, and substituting equations (14a) and (14b), equations (13a), (13b) and (13c) become.

$$
\frac{\partial N_{x}}{\partial x}+\frac{\partial N_{x y}}{\partial y}=0
$$




$$
\begin{gathered}
\frac{\partial N_{x y}}{\partial x}+\frac{\partial N_{y}}{\partial y}=0 \\
\frac{\partial^{2} M_{x}}{\partial x^{2}}+2 \frac{\partial^{2} M_{x y}}{\partial x \partial y}+\frac{\partial^{2} M_{y}}{\partial y^{2}}+N_{x} \frac{\partial^{2} w_{0}}{\partial x^{2}}+2 N_{x y} \frac{\partial^{2} w_{0}}{\partial x \partial y}+N_{y} \frac{\partial^{2} w_{0}}{\partial y^{2}}+q=0
\end{gathered}
$$

Displacement field expression has the following form:

$$
\begin{gathered}
u_{0}=u_{o}^{i}+\lambda u_{0} \\
v_{0}=v_{o}^{i}+\lambda v_{0} \\
w_{0}=w_{o}^{i}+\lambda w_{0}
\end{gathered}
$$

Where: $\left(\mathrm{u}_{0}^{\mathrm{i}}, \mathrm{v}_{0}^{\mathrm{i}}, \mathrm{w}_{0}^{\mathrm{i}}\right)$ : is the displacement field before instability

$\left(\mathrm{u}_{0}, \mathrm{v}_{0}, \mathrm{w}_{0}\right)$ : is any possible displacement field (satisfying boundary conditions and continuity conditions)

$\lambda$ : is an infinitely small scalar regardless of coordinates of the surveyed point.

The phenomenon of instability is seen as a process of producing an extremely small deviation from a balanced position.

Combining (16) and (11), the following relation is formed:

$$
\left\{\begin{array}{l}
N=A \varepsilon^{i}+B k^{i}+\lambda(A \varepsilon+B k)=N^{i}+\lambda N \\
M=B \varepsilon^{i}+D k^{i}+\lambda(B \varepsilon+D k)=M^{i}+\lambda M
\end{array}\right.
$$

Put (16) and (17) into equation (15c) to get a first order equation of $\lambda$, ignoring the second order terms of $\lambda$. This equation is satisfied for all $\lambda$, if the terms of $\lambda$ null out, we have:

$$
\begin{array}{r}
\frac{\partial^{2} M_{x}^{i}}{\partial x^{2}}+2 \frac{\partial^{2} M_{x y}^{i}}{\partial x \partial y}+\frac{\partial^{2} M_{y}^{i}}{\partial y^{2}}+N_{x}^{i} \frac{\partial^{2} w_{0}^{i}}{\partial x^{2}}+2 N_{x y}^{i} \frac{\partial^{2} w_{0}^{i}}{\partial x \partial y}+N_{y}^{i} \frac{\partial^{2} w_{0}^{i}}{\partial y^{2}}+q^{i}=0 \\
\frac{\partial^{2} M_{x}}{\partial x^{2}}+2 \frac{\partial^{2} M_{x y}}{\partial x \partial y}+\frac{\partial^{2} M_{y}}{\partial y^{2}}+N_{x}^{i} \frac{\partial^{2} w_{0}}{\partial x^{2}}+N_{x} \frac{\partial^{2} w_{0}^{i}}{\partial x^{2}} \\
+2 N_{x y}^{i} \frac{\partial^{2} w_{0}}{\partial x \partial y}+2 N_{x y} \frac{\partial^{2} w_{0}^{i}}{\partial x \partial y}+N_{y}^{i} \frac{\partial^{2} w_{0}}{\partial y^{2}}+N_{y} \frac{\partial^{2} w_{0}^{i}}{\partial y^{2}}+q=0
\end{array}
$$

Equation (18) coincides with equation (15c) allowing the determination of elastic configuration (initial configuration) in the case of large horizontal deformation. This equation is not completely linear; however, for simplicity's sake, we use linear theory when determining elastic configuration.

Since $w_{0}^{i}$ is pretty small, the curvature terms of the bending-bearing plate in the equation (19) are omitted. Then this equation becomes.

$$
\frac{\partial^{2} M_{x}}{\partial x^{2}}+2 \frac{\partial^{2} M_{x y}}{\partial x \partial y}+\frac{\partial^{2} M_{y}}{\partial y^{2}}+N_{x}^{i} \frac{\partial^{2} w_{0}}{\partial x^{2}}+2 N_{x y}^{i} \frac{\partial^{2} w_{0}}{\partial x \partial y}+N_{y}^{i} \frac{\partial^{2} w_{0}}{\partial y^{2}}+q=0
$$

Equation (20) coincides with equation (15c) allowing the determination of elastic configuration in the case of small horizontal displacement.

Put the equation (10) into $(15 \mathrm{a} ; 15 \mathrm{~b} ; 15 \mathrm{c})$, the following three basic expressions are achieved: 


$$
\begin{aligned}
& A_{11} \frac{\partial^{2} u_{0}}{\partial x^{2}}+2 A_{16} \frac{\partial^{2} u_{0}}{\partial x \partial y}+A_{66} \frac{\partial^{2} u_{0}}{\partial y^{2}}+A_{16} \frac{\partial^{2} v_{0}}{\partial x^{2}}+\left(A_{12}+A_{66}\right) \frac{\partial^{2} v_{0}}{\partial x \partial y}+A_{26} \frac{\partial^{2} v_{0}}{\partial y^{2}} \\
& -B_{11} \frac{\partial^{3} w_{0}}{\partial x^{3}}-3 B_{16} \frac{\partial^{3} w_{0}}{\partial x^{2} \partial y}-\left(B_{12}+2 B_{66}\right) \frac{\partial^{3} w_{0}}{\partial x \partial y^{2}}-B_{26} \frac{\partial^{3} w_{0}}{\partial y^{3}}=0 \\
& A_{16} \frac{\partial^{2} u_{0}}{\partial x^{2}}+\left(A_{12}+A_{66}\right) \frac{\partial^{2} u_{0}}{\partial x \partial y}+A_{26} \frac{\partial^{2} u_{0}}{\partial y^{2}}+A_{66} \frac{\partial^{2} v_{0}}{\partial x^{2}}+2 A_{26} \frac{\partial^{2} v_{0}}{\partial x \partial y}+A_{22} \frac{\partial^{2} v_{0}}{\partial y^{2}} \\
& \left.-B_{16} \frac{\partial^{3} w_{0}}{\partial x^{3}}-\left(B_{12}+2 B_{66}\right) \frac{\partial^{3} w_{0}}{\partial x^{2} \partial y}-3 B_{26}\right) \frac{\partial^{3} w_{0}}{\partial x \partial y^{2}}-B_{22} \frac{\partial^{3} w_{0}}{\partial y^{3}}=0 \\
& D_{11} \frac{\partial^{4} w_{0}}{\partial x^{4}}+4 D_{16} \frac{\partial^{4} w_{0}}{\partial x^{3} \partial y}+2\left(D_{12}+2 D_{66}\right) \frac{\partial^{4} w_{0}}{\partial x^{2} \partial y^{2}}+4 D_{26} \frac{\partial^{4} w_{0}}{\partial x \partial y^{3}}+D_{22} \frac{\partial^{4} w_{0}}{\partial y^{4}} \\
& -B_{11} \frac{\partial^{3} u_{0}}{\partial x^{3}}-3 B_{16} \frac{\partial^{3} u_{0}}{\partial x^{2} \partial y}-\left(B_{12}+2 B_{66}\right) \frac{\partial^{3} u_{0}}{\partial x \partial y^{2}}-B_{26} \frac{\partial^{3} u_{0}}{\partial y^{3}}-B_{16} \frac{\partial^{3} v_{0}}{\partial x^{3}} \\
& -\left(B_{12}+2 B_{66}\right) \frac{\partial^{3} v_{0}}{\partial x^{2} \partial y}-3 B_{26} \frac{\partial^{3} v_{0}}{\partial x \partial y^{2}}-B_{22} \frac{\partial^{3} v_{0}}{\partial y^{3}}=N_{x} \frac{\partial^{2} w_{0}}{\partial x^{2}}+2 N_{x y} \frac{\partial^{2} w_{0}}{\partial x \partial y}+N_{y} \frac{\partial^{2} w_{0}}{\partial y^{2}}+q
\end{aligned}
$$

In the case of symmetric multi-layer plates $\left(B_{i j}=0\right)$ membrane equations are separate from bending equations, and for pure bending cases: $u_{0}=v_{0}=0$, equations (21a) and (21b) null out. Then the equations $(21 \mathrm{a}, 21 \mathrm{~b}, 21 \mathrm{c})$ are written in the following form:

$$
\begin{aligned}
D_{11} \frac{\partial^{4} w_{0}}{\partial \mathrm{x}^{4}}+4 \mathrm{D}_{16} \frac{\partial^{4} w_{0}}{\partial \mathrm{x}^{3} \partial \mathrm{y}} & +2\left(D_{12}+2 D_{66}\right) \frac{\partial^{4} w_{0}}{\partial x^{2} \partial y^{2}}+4 D_{26} \frac{\partial^{4} w_{0}}{\partial x \partial y^{3}}+D_{22} \frac{\partial^{4} w_{0}}{\partial \mathrm{y}^{4}} \\
= & N_{x} \frac{\partial^{2} w_{0}}{\partial x^{2}}+2 N_{x y} \frac{\partial^{2} w_{0}}{\partial x \partial y}+N_{y} \frac{\partial^{2} w_{0}}{\partial y^{2}}+q
\end{aligned}
$$

For square-layer plate $\left(D_{16}=D_{26}=0\right)$ the equation (22) becomes:

$$
\begin{aligned}
D_{11} \frac{\partial^{4} w_{0}}{\partial \mathrm{x}^{4}}+2\left(D_{12}\right. & \left.+2 D_{66}\right) \frac{\partial^{4} w_{0}}{\partial x^{2} \partial y^{2}}+D_{22} \frac{\partial^{4} w_{0}}{\partial \mathrm{y}^{4}} \\
& =N_{x} \frac{\partial^{2} w_{0}}{\partial x^{2}}+2 N_{x y} \frac{\partial^{2} w_{0}}{\partial x \partial y}+N_{y} \frac{\partial^{2} w_{0}}{\partial y^{2}}+q
\end{aligned}
$$

The equation (23) is also the buckling equation of orthotropic plates. 